\title{
The ITER disruption mitigation trigger: developing its preliminary design.
}

\author{
G. Pautasso ${ }^{1}$, P.C. de Vries ${ }^{2}$, D. Humphreys ${ }^{3}$, M. Lehnen ${ }^{2}$, C. Rapson ${ }^{1}$, G. Raupp ${ }^{1}$, \\ J.A. Snipes ${ }^{2}$,W. Treutterer ${ }^{1}$, A. Vergara-Fernandez ${ }^{2}$, L. Zabeo ${ }^{2}$
}

[1] Max-Plank-Institute für Plasma Physik, D-85748, Garching, Germany

[2] ITER Organization, Route de Vinon sur Verdon, 13067 St Paul Lez Durance, France

[3] General Atomics, PO Box 85608, San Diego, CA 92186-5608, USA

E-mail contact of main author: gap@ipp.mpg.de

\begin{abstract}
.
A concept for the generation of the trigger for the ITER disruption mitigation system is described in this paper. Issuing the trigger will be the result of a complex decision, taken by the plasma control system or by the central interlock system, that the plasma is unavoidably going to disrupt - or has disrupted - and that it requires a fast mitigated shut-down. Given the redundancy of the mitigation system, the plasma control system must also formulate an injection scheme and specify when and how the injectors of the mitigation system should be activated. The parameters and the algorithms required for the configuration and generation of the trigger are discussed.
\end{abstract}

\section{Introduction}

A major disruption is a global instability that terminates the plasma discharge in a tokamak, typically in an uncontrollable way. Once it has happened, the phenomenon can be easily identified, since it coincides with the loss of the thermal energy, followed by the increase of the plasma resistivity and by the ohmic dissipation of the plasma current. Typically, the major disruption of elongated plasmas leads also to the loss of the vertical stability and to the vertical displacement of the whole plasma column, on the resistive time scale of the vessel stabilizing structures. The thermal and magnetic energies lost by the plasma are deposited onto the plasma facing components of the device. In addition, the movement of a plasma carrying current and the decay of the plasma current induce large currents and associated forces in the tokamak conductors.

Plasma disruptions, occurring above a given plasma current and thermal energy, will have to be mitigated in ITER in order to reduce thermal and mechanical stresses on the machine components and prevent their damage [1]. The premises for the mitigation of tokamak disruptions are, firstly, the capability of recognizing that a disruption is going to occur, and secondly, the existence of a mitigation method and the technical feasibility of a mitigation system. Methods of disruption prediction and disruption mitigation are 
currently subject to R\&D on existing tokamaks and provide the know-how for the design of the ITER Disruption Mitigation System (DMS) [2] and of the DMS trigger concept. The massive injection of material (mostly noble gases in the gaseous or frozen state) has been shown to be suitable for disruption mitigation in most of the existing tokamaks [3]. The ITER DMS will also exploit impurity injection; it will consist of a number of gas and pellet injectors and is currently being designed.

This paper concentrates on the problems of recognizing that a plasma disruption is unavoidably going to occur, or it has occurred, and presents the preliminary design of the trigger to the ITER DMS. The related work has been carried out during the preliminary design phase (2014-2016) of the ITER Plasma Control System (PCS) in the framework of a collaboration between the ITER team and a consortium of external institutions [4]. The ultimate purpose of the design presented in the following was - and is - to configure the PCS and its auxiliary systems to properly deal with plasma disruptions. A disruption - also a mitigated one - is an off-normal operation event that could drive various plant actuators to their limits, in which case the PCS must generate appropriate control responses.

The possibility of recognizing that a disruption is going to occur, or has occurred, relies on the know-how on disruption prediction and detection acquired on existing tokamaks: the physics basis for the prediction and detection of terminal plasma instabilities is briefly discussed in section 2. Section 3 of the paper describes and substantiates a concept for the DMS trigger generation and the role of the systems involved. The reliability requirements for the DMS trigger are discussed in section 4. Section 5 outlines the development of the DMS trigger generation function.

\section{Physics basis for disruption prediction and detec- tion}

\subsection{Phenomenology}

The text-book disruption [5] is a phenomenon consisting of two clear phases, that is a fast thermal quench (TQ), during which the thermal energy is lost by the plasma on the millisecond timescale, and the current quench (CQ), during which the toroidal current decays on the inductive-resistive time-scale of the plasma torus. A fast TQ is believed to be the consequence of the growth of MHD modes, which essentially destroy part of the magnetic flux surfaces, by creating magnetic field lines, which fill the whole plasma in a stochastic manner.

In recent experiments in tokamaks with metal walls (JET [6] and AUG), a fast TQ is not always detectable before the $\mathrm{CQ}$, since impurity accumulation and strong core radiation 
can dissipate the thermal energy on the timescale of the energy confinement time, without causing an immediate major disruption. Typically, such plasmas are subject to minor disruptions and loss of vertical stability, which results in a terminal CQ.

Moreover, the plasma may lose its vertical stability without the initial TQ and CQ if its parameters (e.g. current profile, plasma shape, magnitude of disturbances) are outside of the controllability region for the given control system. Such a hot VDE is then followed by a TQ and a CQ.

From an energy balance point of view, during a disruption, the large ohmic- and other types of auxiliary- input power cannot sustain the plasma temperature because of the extremely poor plasma confinement properties, due to anomalously large perpendicular conduction (MHD modes), large quasi-parallel conduction in a stochastic magnetic configuration, a too large impurity density or/and a strong interaction with the PFCs. The consequence of the loss of thermal energy is the cooling of the plasma down to temperatures of the order of $1-100 \mathrm{eV}$, the decay of the plasma current, the recombination of the plasma into neutral gas and the off-normal plasma discharge termination. The Tokamak Physics Bases [7] foresee a TQ duration of $\tilde{1} \mathrm{~ms}$ and a natural CQ lasting longer than $35 \mathrm{~ms}$.

Runaway electrons (REs) are expected to be generated during an ITER CQ, because of the large poloidal flux associated with the toroidal current and the huge avalanche amplification of any small RE seed population [7]. REs can also be generated during the current ramp up in case the toroidal electric field exceeds the critical electric field (e.g. in case of low electron density and large plasma resistivity).

A disruption is preceded by a precursor phase, in which the plasma transits from a more to a less stable state and several plasma parameters evolve in time. Disruption detection and prediction turn out to be possible by following the plasma state evolution.

The next two sections are dedicated to

- the discussion of methods and criteria, that allow predicting that a plasma disruption is going to occur with high probability (section 2.2);

- the detection of the TQ, of the CQ, of loss of vertical control (LVC) and of REs (section 2.3).

\subsection{Disruption prediction}

The tokamak disruption is usually preceded by a phase in which the plasma evolves from a more to a less stable configuration and MHD instabilities, in particular tearing modes and/or kink-like modes, develop. Several mechanisms, leading to disruptions, have been identified and studied, e.g. high density and high beta limits, locked mode in low density plasmas and $q_{95} \sim 2$. Nevertheless, these mechanisms do not cover all the possible 
paths to a disruption. In fact, the plasma parameter regions, where disruptions can happen, are not yet completely well defined. The causes and mechanisms leading to the plasma termination can be very different, but the phase preceding the disruption seems to be characterized by the presence of large MHD modes [8] and typically (but there are exceptions among discharges reaching the beta limit) small thermal confinement time.

\subsubsection{Physics based methods}

The recent work described in reference [9] explores the hypothesis that a plasma disruption occurs when a $(\mathrm{m}, \mathrm{n})=(2,1)$ island, mostly locked, has reached a critical width. Data from three existing tokamaks - i.e. JET, ASDEX Upgrade and Compass - seem to suggest that this is the case; a scaling law was derived for the magnitude of the radial magnetic field, $B_{r}$, measured by coils located outside of the plasma, just before the onset of the TQ. This work represents, at least in part, the physics basis for disruption prediction and future work will be required to confirm its solidity.

It is already clear from the data and from the derived scaling law, that locked modes in plasmas with large $q_{95}$ and low internal inductance generate small values of $B_{r}$ at the coil location and that this value could be just above the noise level. In these cases, the detection of disruption precursors and the monitoring of specific plasma parameters could contribute to the formulation of the disruption alarm. Plasma parameters that are known to change before the disruption and have discriminating capability are: the internal inductance, the radiated power normalized to the input power and the radiation profile, the plasma beta (or energy) and its time derivative, the loop voltage and the confinement time, among others. The recent analysis of a DIII-D $(\mathrm{m} / \mathrm{n})=(2 / 1)$ initially rotating locked mode database [10] shows that $l_{i} / q_{95}$ and $d_{\text {edge }}$ (i.e. the shortest distance between the $2 / 1$ island and the plasma separatrix) can discriminate well between disruptive and nondisruptive initially rotating $(2,1)$ modes.

A universal algorithm for disruption prediction does not exist yet. The whole topic of integrated plasma control and disruption prediction requires more attention in terms of experimental time, data analysis and concept development. ITER will profit from work of development of such an algorithm on existing tokamaks. Real-time MHD (linear and non-linear) stability calculations and evaluation of the plasma evolution through modelling, presently not yet developed enough to be reliable, could become useful tools for the detection of unstable plasma states at the time ITER will go into operation.

\subsubsection{Supervised methods}

Many published works, aimed at developing methods of disruption prediction, make use of a large database of plasma parameters, from both stable plasma phases and pre-disruption phases, and rely on statistical analysis to derive, from the database, the probability that a 
disruption will happen, within a time interval (given by the duration of the pre-disruption phase used to build the database) and in a given region of the plasma operational space. This probability is a function of the multi-D parameter space and allows assigning a disruption probability (prediction alias output function) to every new plasma state input to the function. The general finding of all these works is that disruptions can be predicted with a high probability (of the order of 80-90\%) but not with a certainty of $100 \%$. See Section 7.4 of [11] for a short but critical review of these methods.

Although they can exhibit a high prediction rate and low false alarm rate when optimized, these methods have several disadvantages. In particular,

- they need a database of examples on the basis of which an algorithm is derived, and therefore they cannot be used from the start of machine operation;

- they cannot deliver a reliable output outside of the plasma region on which the algorithm was derived - i.e. they cannot extrapolate - and generally

- they do not contain all of the plasma parameters needed to describe the physics of the disruptive boundary, and, therefore, they age, e.g. they become invalid as the plasma operation evolves.

\subsubsection{Plasma control and disruption prediction}

Several specialized PCS modules will take care of plasma control, and in particular of MHD control, in ITER. Some of these modules will presumably be dedicated to a specific plasma parameter region or boundary in which MHD instability leading to disruption may occur. These control modules could and should also be designed to fulfill the disruption prediction task. The modules controlling ramp-up and ramp-down (current profile control; locked mode avoidance), plasma position (vertical, radial and gaps plasma-wall), density, radiation, impurity accumulation, neoclassical tearing modes (NTM), beta and resistive wall modes, should have enough information (diagnostics, knowledge on the controllable plasma range, status of actuators, etc.) at their disposal, to be able to generate a loss-of-control signal. For example, the NTM control module should have all the information for the detection and control of tearing modes (presence, frequency, amplitude, location from magnetic probes, ECE and/or Soft X-Ray, ECRH power in input and still available, control scheme and controllability range) and know when the mode is growing. The disruption prediction task will consist in recognizing that the plasma-machine status is outside of the controllable parameter region. The specific loss-of-control signal could be just one of a long list of signals, which indicate that the plasma-machine status is close to the disruption instability. It is beyond the scope of this work to discuss how the different parts of the PCS should share this type of information. 


\subsection{Disruption detection}

The TQ, the CQ, the LVC and the RE detection algorithms for ITER are expected to be similar to the algorithms which are used - or could be used - in present tokamaks, and will be developed during the course of operation. The table in fig. 6 summarizes diagnostics and criteria for the detection of these phenomena. These must be identified by the PCS because they prompt the plant system shut down and determine the type of disruption mitigation scheme, in case mitigation is required.

\subsubsection{Thermal quench}

Examples of detection of the TQ, for plasma control purposes in present tokamaks, are not known to the authors. Nevertheless, a sudden loss of thermal confinement, i.e. a sudden drop of the electron temperature - over the whole plasma cross-section and, particularly in the plasma core - or of the thermal energy, can be detected if the temperature or the energy measurements are correct (e.g. if the ECE temperature measurements are not in cut-off) and if the drop is larger than the noise/error level. In addition, the temperature/energy drop must be larger than values typical of other - non-terminal MHD phenomena, e.g. ELMs and sawteeth.

\subsubsection{Current quench}

The parameter necessary for the detection of the current quench is the plasma current, which is known to the PCS in real time, since it must be controlled. The CQ in existing tokamaks is routinely detected for the following reasons and in the following ways:

- in real time, by the PCS, since this system must know when the plasma discharge ends and when to initiate the plant shut-down.

- In this case, the CQ detection can occur relatively late during the current decay, and a criterion of the sort $\left|I_{p}-I_{p, r e f .}\right|>\Delta I_{p, C Q}$ is appropriate. $I_{p, r e f}$ is a pre-programmed reference current known to the PCS and $\Delta I_{p, C Q}$ is a threshold value.

- Detecting $\left|d I_{p} / d t\right|<\left|d I_{p} / d t_{C Q}\right|$ is an alternative method, where the time derivative must be intended as finite difference over a given time interval. $\left|d I_{p} / d t_{C Q}\right|$ is an appropriate threshold value known to the PCS;

- off-line, for data analysis. The CQ is easy to identify once it has happened.

- The detection of the beginning of the current quench can be based on $\left|d I_{p} / d t\right|>$ $d I_{p} / d t_{C Q o n s e t}$ (where $d I_{p} / d t_{C Q o n s e t}$ is a threshold value known to the PCS). The whole $I_{p}$ time history can be used to confirm the time of CQ onset. 
- The correlation of the previous condition with a sudden drop in the temperature/energy (TQ), and/or with a sudden drop of the plasma internal inductance could improve the detection reliability.

- The off-line detection of an advanced current quench can also employ the real-time algorithm mentioned above.

\subsubsection{LVC}

A large deviation of the vertical plasma position from the reference value is a good indicator that the position control is not being maintained by the PCS. A criterion of this type is used in present machines, e.g. ASDEX Upgrade [12], to detect the LVC. The maximum allowed deviation depends on the actual plasma and PCS parameters.

LVC can in theory be predicted by computing the stability margin of the given plasma and its maximum controllable displacement (see e.g. [13]).

\subsubsection{Runaway electrons}

REs can be detected since they generate anomalously large ECE, Soft X-Ray, Hard X-ray emission, synchrotron and Bremsstrahlung radiation.

\section{The DMS trigger: a concept}

\subsection{The disruption mitigation function}

The necessary conditions for the fulfillment of - what is called here - the disruption mitigation function (DMF) are, firstly, the capability of recognizing that a terminal instability is going to cause large thermal loads and/or forces and needs mitigation, and secondly, the existence of an effective mitigation system. Therefore, the DMF consists of two main sub-functions, namely

1. deciding that the plasma has to be shut-down with the DMS and issuing a trigger decision (see section 3.3.8); formulating the DMS injection scheme, which specifies how the DMS should be triggered (e.g. which injector should be fired and when, see section 3.3.7);

2. executing the DMF, according to the actual injection scheme, by activating the DMS.

Fig. 4 shows a schematic representation of the processes and systems involved in fulfilling the DMF. This article concentrates on the first sub-function, which is called the DMS 
trigger generation function in the following. The term DMS trigger indicates an array of signals, carrying the information that given DMS injectors must be fired at a given time (fig. 1).

\subsection{Systems involved}

The DMF is assigned in ITER to a cluster of systems (fig. 2) consisting of the Central Interlock System (CIS), the PCS and the DMS, interacting with the plant system (PS, e.g. coils, vacuum, heating, fuelling and other systems) and with the plasma. The different systems involved are in an advanced design phase and the functional interfaces among them are being discussed and established.

\subsubsection{The role of the CIS}

The CIS [14] is responsible for the protection of several ITER systems (e.g. the cryogenic system, the magnets and their associated equipment) and executes automatic interlocks generated on the basis of the plant system status or operational limits (e.g. plasma current exceeding a maximum value). Among the systems involved in executing the DMF, the CIS is the only system actively triggering the DMS injectors and consequently it has several responsibilities:

- it must be able to accept and store/update the DMS injection scheme provided by the PCS,

- it may issue a trigger decision, independently of the PCS, and trigger the DMS, according to the known injection scheme, as an ultimate tool to prevent plant system damage;

- it must be able to accept a trigger decision from the PCS and trigger the DMS;

- it activates each individual DMS injector according to the injection scheme provided by the PCS;

- it initiates a fast shut-down with the DMS when the plasma current exceeds a given value;

- it informs the plant systems, that need to be protected, when the DMS is triggered;

- in case of failure of the PCS, the CIS takes over the responsibilities of triggering the DMS according to a valid DMS injection scheme and of shutting down plasma and PS according to a predefined procedure. 


\subsubsection{The role of the PCS}

The PCS is responsible for the control of the plasma during normal and off-normal operation and technical pulses. The main roles of the PCS, in the case of an irreversibly unstable plasma should be:

- recognizing on the basis of given criteria and plasma parameter measurements that the plasma has become irreversibly unstable; recognizing that vital plant subsystems have become unavailable and a plasma disruption is imminent;

- deciding whether a fast mitigated plasma shut-down must be initiated, on the basis of the magnitude of the disruption effects, and eventually issue a trigger decision;

- deciding which DMS injector(s) must be triggered and at which time, i.e. generate the DMS injection scheme;

- sending periodically to CIS an updated DMS injection scheme;

- assisting CIS in investment protection, i.e. in bringing the plant systems to a safe state in case of a plasma disruption and plant shut down;

- recognizing that REs are present during the discharge; deciding either to suppress them (e.g. by increasing the electron density) or to terminate the discharge, either in a controlled way or via DMS;

- recognizing on the basis of plasma and DMS parameters that the DMS availability is not sufficient to mitigate a disruption with the actual plasma parameters (one or more injectors not "ready"; leak detected etc.); recognize that the communication of data from the DMS is interrupted; order a preventive controlled and slow plasma ramp down in these cases.

\subsubsection{The role of the DMS}

The DMS is designed to consist of a number of gas and pellet injectors; it will exploit impurity injection and is currently being designed [1], [2]. The DMS executes the DMF by injecting impurities into the plasma. In detail, it

- shall terminate the plasma, to limit disruption impact on the first wall and mechanical structures, resulting from disruptions;

- receives trigger instructions from CIS and executes them; it must operate a set of injectors (gas valves or pellet injectors) for TQ and CQ mitigation and RE suppression; 
- must monitor the injectors and related system component status and inform the PCS (directly or through CIS) about this status.

\subsection{The DMS trigger generation function}

\subsubsection{The output}

The ultimate output (and scope) of the overall DMS trigger generation function (see fig. 3) is an array of signals, namely a signal for each DMS injector, which carries the information that the injector has to be fired at a given time. This output will be called here the DMS trigger. The DMS trigger is issued as the result of a trigger decision, which can be taken either by the PCS or by the CIS (section 3.3.8), and is configured on the basis of the DMS injection scheme generated by the PCS.

\subsubsection{The input}

The generation of the DMS trigger requires processing sets of real time inputs with appropriate algorithms. Four classes of real time inputs and related algorithms have been identified for this purpose and are defined in the following. Two classes of events, namely

(I) the prediction (= forecast with a large probability that a phenomenon will occur) and the detection (= phenomenon is occurring or has occurred) of terminal plasma instabilities and

(II) the prediction and the detection of plant system faults, potentially leading to disruptions,

could require the intervention of the DMS for a fast and mitigated plasma shut-down. Additional information about

(III) the region of the plasma parameter space, in which the expected mechanical and thermal effect of a disruption on the machine components must be mitigated; and about

(IV) the status of the DMS injectors

are then required for configuring and issuing the DMS trigger.

\subsubsection{Disruption prediction and detection (I)}

The terminal plasma instabilities, which must be predicted and detected are: 
- a plasma status with high probability of disruption (i.e. an unavoidable disruption) and with the immediate need for fast and mitigated shut-down; the decision is taken by the PCS on the basis of one or more precursors, with an appropriate algorithm, or taken by a plasma control algorithm;

- the fast thermal quench (TQ);

- the current quench (CQ);

- the loss of vertical control (LVC);

- the presence of runaway electrons (REs).

The reason why all these phenomena should be detected, is because the DMS mitigation scheme (e.g. the type and amount of impurities to be injected) might depend on the plasma status and on the disruption phase predicted and detected. For example, the detection of an advanced $\mathrm{CQ}$ and the prediction of RE generation would call for the injection of high $\mathrm{Z}$ gas for the collisional dissipation of the REs.

\subsubsection{Plant faults (II)}

Plant system faults, that could potentially lead to a disruption, will be detected by the PCS, which can decide to send a request to the CIS to initiate disruption mitigation. Eventually, the class-I events will follow these plant faults. The reason why these plant faults - leading to a disruption - should be detected, is because they allow gaining time, to attempt a fast but controlled shut-down, and/or to increase the disruption prediction reliability.

\subsubsection{Plasma parameter region requiring mitigation (III)}

Plasmas with large current and/or thermal energy will require disruption mitigation. Parameters other than current and energy could quantitatively refine the evaluation of the expected disruption effects and the type of mitigation required. In general, several plasma parameters (which will be specified in the course of ITER operation) will define the operational regions, which require disruption mitigation. These regions and the type of mitigation will have to be specified by an algorithm (e.g. a mapping function or look-up table, with plasma parameters in input and "mitigation: yes or no, and how" as output) and made available to the PCS.

The algorithm will have to be constructed off-line on the basis of the disruption loads expected if the plasma were disrupting with the momentary plasma parameters, and allowable load limits. In particular 
- the estimated maximum force on selected components (e.g. vessel, divertor structures, blanket modules. They are functions of the toroidal current, the elongation, the triangularity, the magnetic field, the momentary or expected $\left.d I_{p} / d t\right)$ and the respective stress limit;

- the estimated maximum thermal load on divertor or PFCs (it is a function of the thermal energy, the plasma shape, the amount of impurity present in the plasma) and the respective allowable load;

- the expected RE current, energy and heat load

will be needed to define the plasma parameter regions requiring mitigation and the proper scheme.

\subsubsection{DMS injector status (IV)}

A matrix, containing information on each single injector, e.g. whether the injector is ready-to-fire or not, which is the injector type (gas/pellet), the gas species and quantity in the injector, eventually the injector response time, must be available to the PCS for the DMS trigger generation. The PCS must make sure that enough injectors are available, in case a mitigated shut down becomes necessary, and must start a controlled plant shut-down in case of insufficient mitigation capability.

\subsubsection{DMS injection scheme}

The type of event and a set of selected real time plasma parameters - as proxy of the expected magnitude of the disruption loads - form the input of a function (derived on the basis of modelling and experimental verification) relating them to the appropriate injection scheme (see fig. 5). This function will be defined on the basis of the physical understanding, which is being gained with mitigation experiments on existing devices, and fine-tuned with dedicated experiments on ITER (see section 5).

The injection scheme should be allowed to depend on the type of events. For example: Mitigation after the TQ could require another quantity or type of gas than mitigation triggered before the TQ. RE suppression could require an amount of higher $\mathrm{Z}$ material larger than any other event/ instability.

The DMS injection scheme is continuously (with an appropriate time cycle) generated by the PCS, on the basis of the actual plasma status and of the DMS status, and passed over to the CIS. 


\subsubsection{Trigger decision}

The PCS can decide that the DMS must be triggered after detecting a terminal plasma instability (class-I) or a plant fault (class-II). Also the CIS can issue a trigger decision after detecting a fatal plant fault or a loss of current control. The input class-III, which allows to decide whether the actual plasma requires mitigation or not, can be used at this point to block or rectify the trigger decision. In this case, the CIS should have information about the plasma parameter region requiring mitigation. Alternatively, the input class-III can be used by the PCS in generating the DMS injection scheme (i.e. no injector firing if the foreseen unmitigated disruptions loads are tolerable).

When writing this article, the algorithms involved in the DMS trigger generation and the role of the different systems, involved in the fulfillment of the DMF, have not been specified in detail yet.

\subsubsection{From the detection of a terminal instability to DMS firing: an epilogue}

The first two input classes, indicated as I and II, are the detection of plasma events and of plant faults, which, with high probability, lead to a disruption and can require mitigation. The mitigation action will be initiated if-and-only-if the disruption effects are larger than threshold values that could damage machine components. Class-III consists of the plasma parameter set, which identifies the magnitude of the disruption effects; if these plasma parameters may cause loads larger than the allowed mechanical and thermal loads, then the DMS is triggered; otherwise, the trigger is not forwarded to the DMS. Class-IV contains information on the available and appropriate injectors and is used to select them to be fired.

The following is a strawman of the DMS trigger generation algorithm :

Q: Is a terminal instability or a plant fault leading to disruption detected?

If no: continue pulse. Start from $Q$ again.

If yes: is plasma state in region requiring mitigation?

If no: let disruption happen without mitigation;

shut down plant system. STOP.

If yes: choose proper injection scheme;

choose injectors (matrix on injector status);

trigger them;

shut down plant systems. STOP.

\subsubsection{Runaway electrons}

The generation of REs is a serious threat to the ITER PFCs at any relevant plasma current. Therefore, RE control schemes are desirable. Several measures can influence the 
RE beam in existing tokamaks:

- the position control of the RE beam is possible if the plasma elongation is small;

- the OH system can be used to control or, at least, significantly influence the RE current [15];

- the RMP coils have been used in some cases to increase RE losses [16];

- injection of high Z (e.g. argon) gas suppresses the RE current efficiently in medium size tokamaks [15], [17].

Nevertheless, of all these methods, only the last one is presently believed to be routinely applicable to RE suppression in ITER. In addition, the long time interval between triggersent-to-DMS and gas assimilation by the plasma (latency time $>30 \mathrm{~ms}$ ) is of the order of the time interval for the RE beam to develop (a fraction of the CQ duration, i.e. a fraction of 50-150 ms). The natural (without high $\mathrm{Z}$ injection) life time of the RE beam in ITER will depend on the time scale of its vertical/radial movement - not known - and, more in general, on its MHD stability. If the lifetime of the RE beam is of the same order of magnitude of the DMS latency time, the injection of high $\mathrm{Z}$ material must be initiated feed forward, before detecting the presence of RE. RE position/current control, even if it is unlikely to be applicable to most ITER scenarios, should still be envisaged in the PCS design and in the DMS trigger generation function, because it may be occasionally applicable.

\subsection{DMS-fired notification to other systems}

Most of the plant systems must be notified that the DMS has been fired (also that the disruption has occurred) to start the plant shut-down. Plant systems, that must be notified before the trigger is sent to DMS, to protect themselves, have not been identified yet. All ITER systems should be designed to survive without damage or permanent loss of functionality the sudden rise of vessel pressure due to massive material injection. Advantages can be gained in the event-dedicated control of some systems in existing tokamaks; e.g.

- the gas injection system should stop plasma refuelling in case of a density limit;

- the ECRH system should keep heating the island during the DMS latency time;

- the input power should be decreased during the DMS latency time in case of a beta limit; 
- reduction of elongation and current decrease the mechanical load of the impending disruption.

Nevertheless the particle and energy confinement time of ITER are much longer than the DMS latency time (20-30 ms) and it seems unlikely that these measures can influence the plasma behavior in this time interval. Measures to reduce the impact of the disruption effects on the machine should be taken at an earlier time, when an emergency, but relatively slow shut-down (w/o DMS intervention) is initiated.

\section{Reliability of the DMS trigger}

The expected heat loads during the thermal quench will require mitigation action above stored thermal energies of about 25 MJ. Thermal loads during the current quench are expected to become critical above about 5 MA. Avoidance of high electromagnetic loads caused by halo currents will require a high mitigation reliability above about 8 MA [1]. REs could be generated during the CQ at any plasma current and their interaction with the PFCs could cause local melting. Thus, the DMS is needed from early operation on and high reliability of the DMS trigger has to be achieved already during the first experimental phase.

The DMS trigger is called reliable if it induces a mitigation action for all (but only for those) plasmas that cannot be controlled by the PCS, and that would disrupt and cause thermal and mechanical loads above the allowable thresholds. Therefore the DMS trigger "reliability" will depend on different factors:

- on the degree of reliability of the algorithm for disruption prediction (physics criteria known at the time of ITER operation; few/no false alarms; high percentage of disruption predicted correctly and in-time);

- on the degree of optimization of the interaction between different systems signalling loss-of-control;

- on the correctness of the measurements in input to this algorithm;

- on the appropriateness of the measurements in input to this algorithm;

- on the correctness of the DMS status known to the PCS;

- on the degree of optimization of the mapping between disruption loads and the plasma parameter region.

The DMS trigger will require different levels of reliability, depending on the operational phase, that will have to increase along with the increase of the magnitude of the plasma 
current, of the magnetic field, and of the thermal energy. The number of disruptions that should correctly be predicted in each phase of operation depends on many factors. An estimate of this number requires:

- knowing (or assuming) the total number of disruptions;

- knowing (or assuming) a distribution function of the disruptions as a function of plasma parameters;

- knowing/evaluating the magnitude and time history of the mechanical and thermal stresses caused by the single disruption on different conductors and the relative rupture/fatigue limits;

- knowing the thermal erosion of the PFCs and the component lifetime.

The mechanical components, that are expected to undergo stresses above the fatigue limits will have to be instrumented with diagnostics that allow the indirect evaluation (currents and fields) or direct measure of the stresses or forces. The continuous recording of the time dependent loads will allow the estimation of the component lifetime, risk of component failure etc. and a re-evaluation of the allowed unmitigated and mitigated disruptions.

Reference [18] extensively discusses how the impact of disruptions can be accounted for and how it is related to the mitigation success rate.

\section{DMS trigger development}

The DMS trigger concept, presented in section 3, is generic/flexible enough to allow for its development and tuning on the basis of the experience gained on present tokamaks from now to the start of ITER operation and on the experience gained during the operation of ITER.

An intensification of $R \& D$ on disruption prediction (and avoidance) on existing tokamaks in the coming years could already answer several questions, which would consolidate the approach to the ITER disruption prediction concept, e.g. can a disruption be predicted using only magnetic measurements? If not, which other measurements are necessary? Or, what determines the length of a LM phase and how does it scale among devices? How to extrapolate disruption prediction from one plasma parameter space to another, i.e. which are the plasma conditions for a disruption to occur?

ITER will start operating on a limited operational space, at low current and magnetic field, low auxiliary heating and therefore low thermal energy, which will be progressively expanded. The trigger development needs to follow this development and its reliability 
must increase as the magnitude of the disruption loads increase. A number of disruptions will have to occur unmitigated at the beginning of ITER operation, until mitigation will become really necessary, since information on the dynamics of natural disruptions will provide useful information for the definition of the whole mitigation strategy. Particularly

- the evolution of the plasma parameters during the disruption precursor phase,

- the plasma scenarios leading to disruptions,

- the dynamics of the TQ (e.g. many minor disruptions or a major TQ?) and of the CQ,

- the magnitude and time evolution of the related power loads on the PFCs and mechanical forces on the conducting structures in unmitigated disruptions and their comparison with the expected (on the basis of present tokamaks physics known and modelling) evolution

will provide information for tuning the detection of the terminal plasma instabilities and define/confirm the mapping between plasma parameters and disruption loads. The dependence of the DMS mitigation efficiency on impurity species (in gaseous or frozen form), quantities and plasma parameters will have to be assessed/confirmed. For example, the optimal recipe for the DMS injection scheme will be established after dedicated experiments already foreseen in the ITER Research Plan.

The precise definition of the inputs and algorithms to be used for the detection of terminal instability will depend on their dynamics in an ITER plasma. The algorithms will include thresholds and coefficients, which will be determined/fine-tuned with appropriate experiments in ITER.

In the end, the whole disruption prediction concept will need to be tuned to optimize ITER operation. The complexity behind the development of the DMS trigger will strongly depend on the natural disruption dynamics and experimental program. A simpler (with a few tunable parameters) disruption prediction/mitigation scheme will be sufficient during the first operation phases, but could turn out to be less reliable than a more complex scheme, which requires more time for development and will be needed at high plasma performance.

\section{Summary}

A concept for the generation of the ITER DMS trigger has been presented in this paper. The generation of the DMS trigger requires that the PCS be capable of predicting and detecting several terminal plasma instabilities, and deciding, whether or not they require 
mitigation. Disruption prediction algorithms, of different complexity, have been developed on existing tokamaks. They suggest that disruption prediction will be possible in ITER; nevertheless they must be developed further, on the basis of physics understanding of the disruption precursor phase, in order to become portable and reliable. The scaling of the disruption effects with plasma parameters is known - although with some uncertainty -. Therefore the PCS will be able to decide on the basis of a set of rules, whether the terminal instability in the actual plasma must be mitigated or not.

The PCS will also be responsible of formulating a DMS mitigation scheme which describes which injectors will fire and at which time. The DMS mitigation scheme will be a function of DMS, plasma and plant parameters. The physics basis relating the plasma status with the amount/type of impurities, required for effective mitigation, is being derived with appropriate experiments on and simulations of the existing tokamak devices.

The ultimate development of the trigger and the fine tuning of the prediction/detection algorithms will have to occur during ITER operation, on the basis of the experience gained on the device.

\section{Acknowledgements and Disclaimers.}

ITER is the Nuclear Facility INB no. 174. This paper explores new directions for management of disruptions that are not yet introduced into the ITER technical baseline. The nuclear operator is not constrained by the results presented here. The views and opinions expressed herein do not necessarily reflect those of the ITER Organization.

\section{References}

[1] M. Lehnen et al., Journal of Nuclear Material, 463 (2015) 39

[2] L.R. Baylor et al., Disruption Mitigation System Developments and Design for ITER, 25th Fusion Energy Conference, Saint Petersburg, Russia, 13-18 October 2014, contribution FIP/2-1

[3] E.M. Hollmannn et al., Physics of Plasma, 22 (2015) 021802

[4] J.A. Snipes et al., Overview of the Preliminary Design of the ITER Plasma Control System, 26th Fusion Energy Conference, Kyoto, Japan, 17-22 October 2016, contribution EX/P6-36

[5] J. Wesson, Tokamaks, Clarendon Press, 2nd edition, Oxford (1997) pp. 360-361 
[6] M. Lehnen et al., Impact and mitigation of disruptions with the ITER-like wall in JET, 24th Fusion Energy Conference, San Diego, USA 8-13 October 2012, contribution EX/91

[7] T.C. Hender et al., Nuclear Fusion, 47 (2007) 128-202

[8] P.C. De Vries et al., Nuclear Fusion, 51 (2011) 053018

[9] P.C. De Vries et al., Nuclear Fusion, 56 (2016) 026007

[10] R. Sweeney et al., Nuclear Fusion, 57 (2017) 016019

[11] Active Control of Magneto-hydrodynamic Instabilities in Hot Plasmas, Springer Series on Atomic, Optical and Plasma Physics, 2015, Springer-Verlag

[12] Y. Zhang et al., Nuclear Fusion, 51 (2011) 063039

[13] D.A. Humphreys et al., Nuclear Fusion, 49 (2009) 115003

[14] A. Vergara Fernandez et al., Fusion Engineering and Design, 86 (2011) 1137-1140

[15] E.M. Hollmannn et al., Nuclear Fusion, 51 (2011) 103026

[16] M. Lehnen et al., Phys. Rev. Letters, 100 (2008) 255003

[17] G. Pautasso et al., Plasma Physics and Control Fusion, 59 (2017) 014046

[18] M. Lehnen et al., Plasma Disruption Management in ITER, 26th Fusion Energy Conference, Kyoto, Japan, 17-22 October 2016, contribution EX/P639

[19] A. Vergara Fernandez et al., Fusion Engineering and Design, 86 (2011) 1137-1140 


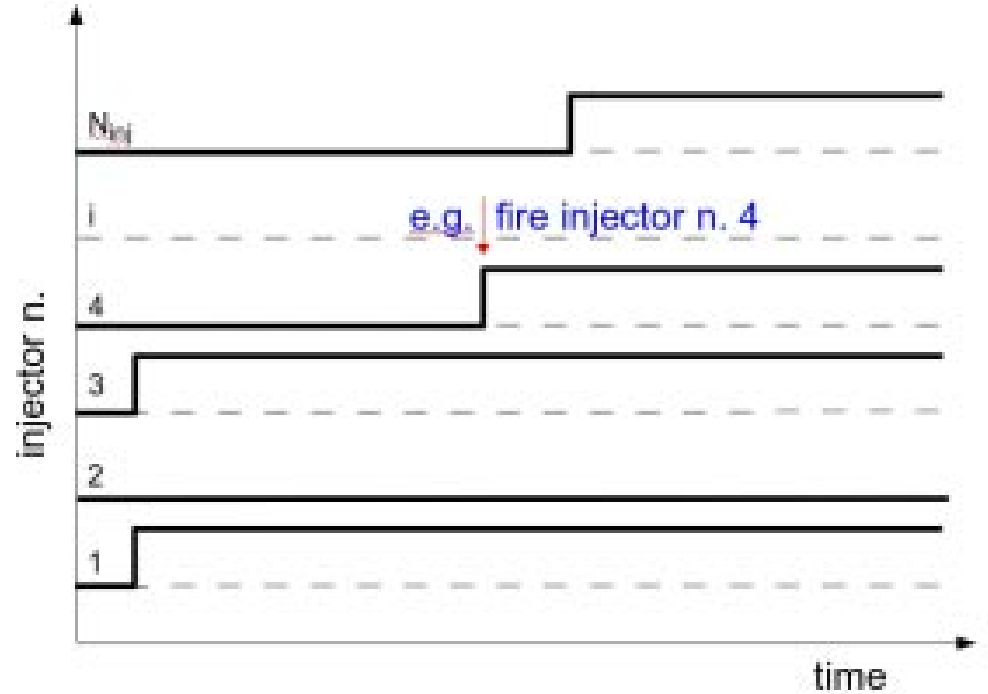

Figure 1: The DMS trigger. 


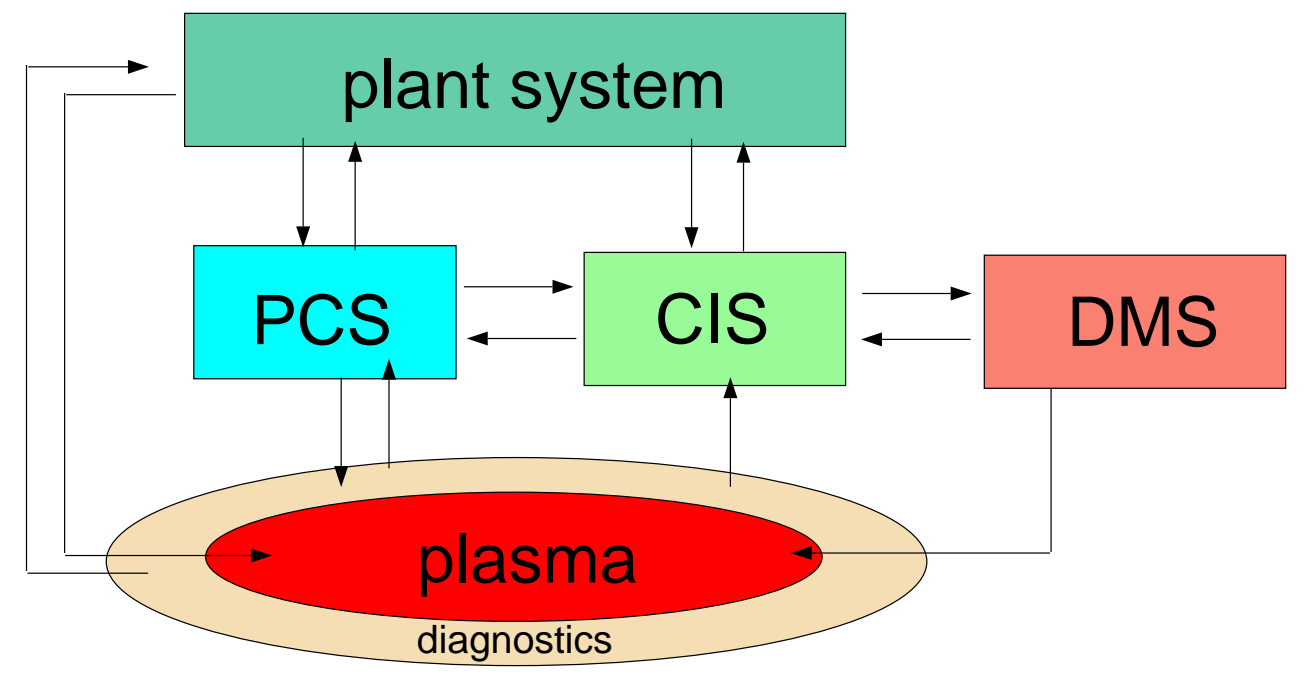

Figure 2: Systems involved in the fulfillment of the disruption mitigation function. 


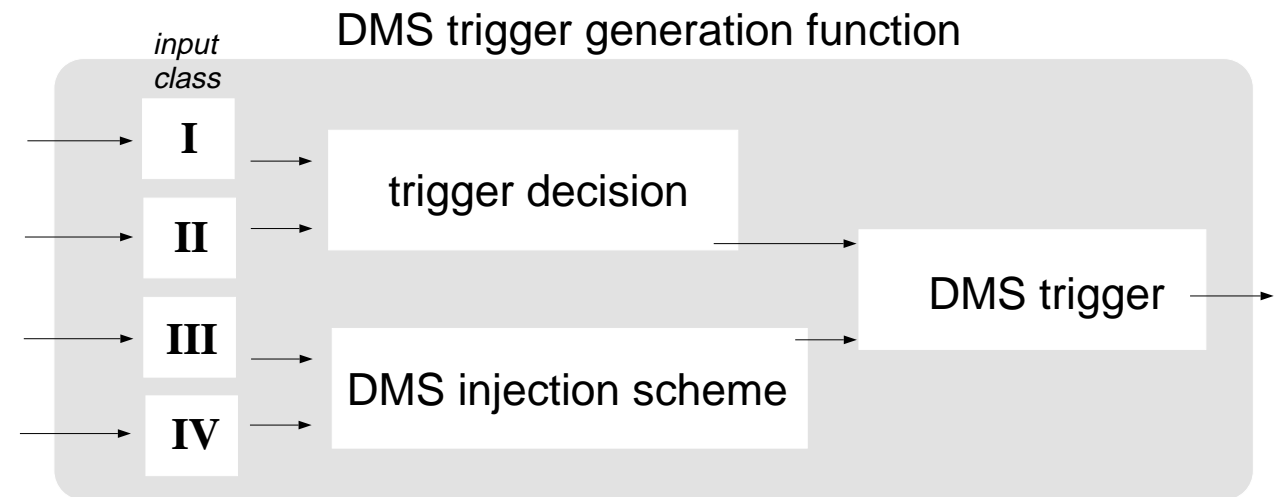

Figure 3: Logical representation of the trigger generation process. 


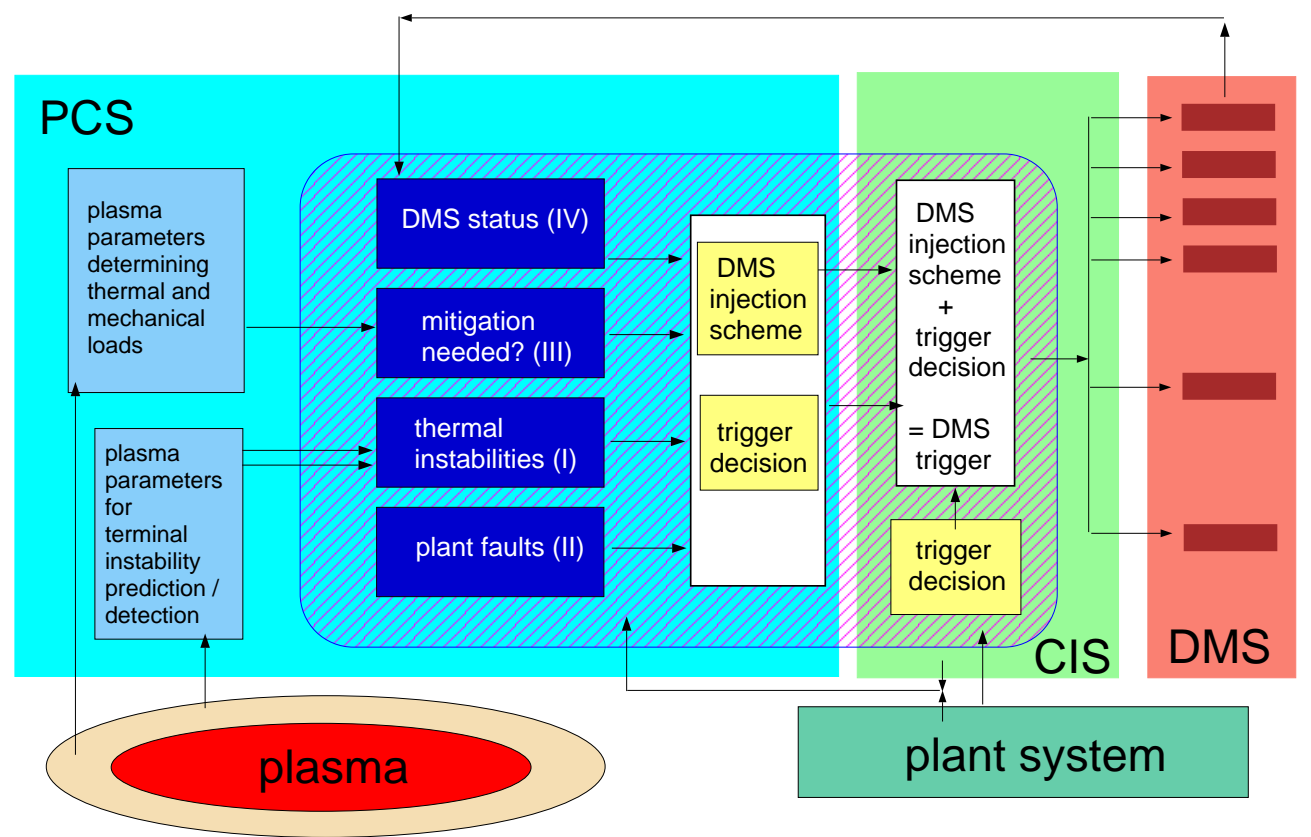

Figure 4: Schematic representation of the DMF. The dark blue boxes are the algorithms processing the four input types described in this section. The yellow box represents the DMS trigger generation function. The brown boxes within the DMS are the individual injectors. 


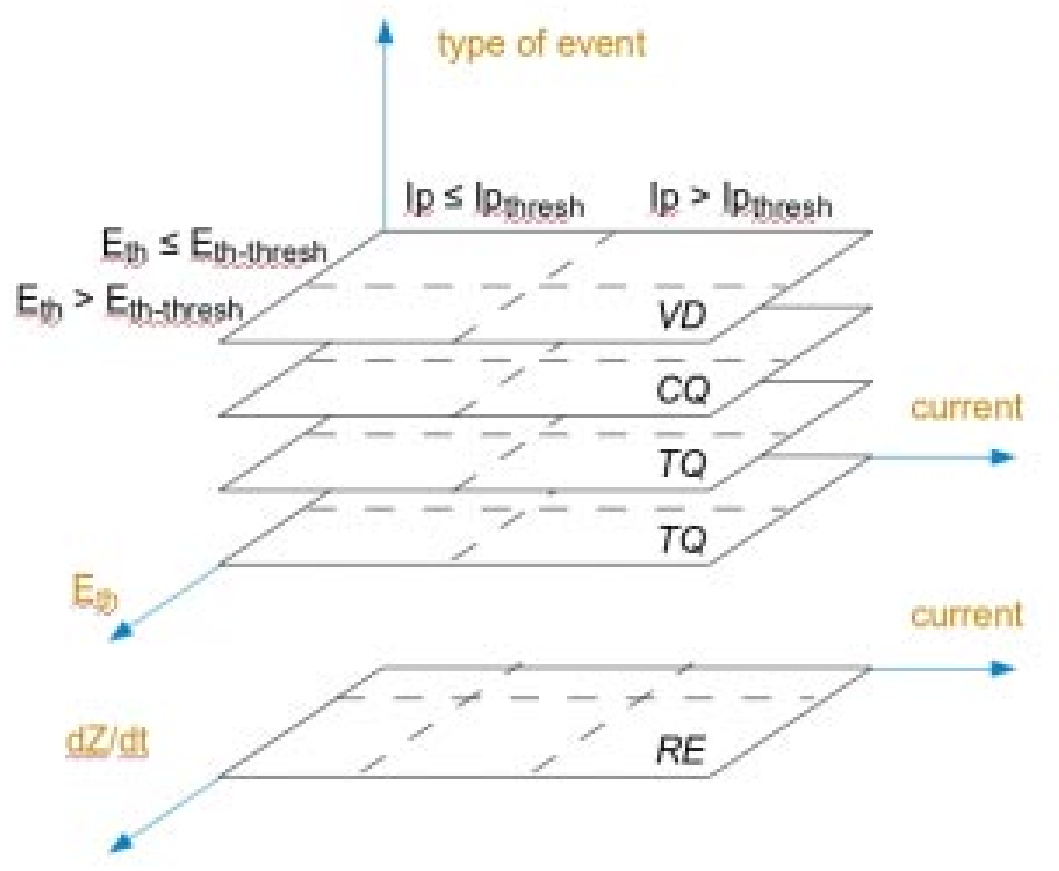

injection scheme generation rules (straw-man)

Figure 5: A graphical representation of the DMS injection scheme generation function. 


\begin{tabular}{|c|c|c|c|}
\hline $\begin{array}{l}\text { Pbene } \\
\text { menoe }\end{array}$ & $\begin{array}{l}\text { Paraneter/ } \\
\text { diagnostic }\end{array}$ & Criterion & Detection reliability \\
\hline TQ & $\begin{array}{l}\text { diamagnetic } \\
\text { loop ot } \\
\text { magnetics for } \\
\text { equilibrimu }\end{array}$ & sudden thermal energy or beta drop & high \\
\hline TQ & \begin{tabular}{|l|} 
election \\
temperature
\end{tabular} & sudden central electrou tempanature drop & high \\
\hline $\begin{array}{l}\text { TQCQ } \\
\text { onset }\end{array}$ & $\begin{array}{l}\text { interual } \\
\text { inductance }\end{array}$ & stadden drop of li (only for layge li) & $\begin{array}{l}\text { high at large li: correlation } \\
\text { with Ip spike }\end{array}$ \\
\hline $\begin{array}{l}\text { CQ } \\
\text { onset }\end{array}$ & toroidal curreat & $|\mathrm{dI} p / \mathrm{dt}|>\mathrm{dlp} / \mathrm{dt}$ ceqeen & very high \\
\hline $\mathrm{CQ}$ & toroidal current & $\mid$ Ip - Iprefarase $\mid>\Delta \mathrm{Ipamad}$ & very high \\
\hline CQ & toroidal curreat & dipidr $<$ dlpidice & very high \\
\hline LVC & $\begin{array}{l}\text { magretics for } \\
\text { equilibrium }\end{array}$ & $\begin{array}{l}\text { deviation of plasta position from } \\
\text { reference value > given threshold }\end{array}$ & very high \\
\hline RE & Bremsstrahling & $\begin{array}{l}\text { radiation enission larger that compatible } \\
\text { with plasma density and temperature }\end{array}$ & high \\
\hline RE & SXR & $\begin{array}{l}\text { radiation emission larger that compatible } \\
\text { with plasma density and temperature }\end{array}$ & high \\
\hline RE & ECE & radiation enaission not due to temperature & high if not in cut-olf \\
\hline RE & HXR & HXR not due to neutrons & high \\
\hline
\end{tabular}

List of criteria for she dewerion of $T Q, C Q, L V C$ and $R E$ s.

Figure 6: List of diagnostics and criteria for the detection of terminal instabilities. 